\title{
I DOVERI GIURIDICI NELL'ORDINAMENTO COSTITUZIONALE ITALIANO
}

\author{
OS DEVERES JURÍDICOS NO ORDENAMENTO CONSTITUCIONAL ITALIANO
}

\section{Giovanni Chiarini}

Avvocato del Foro di Piacenza. Si occupa principalmente di diritto e procedura penale; Già tirocinante ex art. 73 D.L. 69/2013 presso la Procura della Repubblica di Lodi; Si è laureato in diritto costituzionale presso 1'Università degli Studi di Parma ed è autore di varie pubblicazioni giuridiche; Email: chiarini.g@gmail.com

Convidado

\begin{abstract}
Questo breve scritto intende affrontare il concetto di dovere giuridico, analizzandolo in particolare nell'ambito della teoria generale del diritto. Partendo da una breve disamina, astratta e filosofica, di tale significato, si è poi cercato di analizzare il dovere come "dovere costituzionale", con particolare riferimento alla Costituzione Italiana e facendo un breve excursus, anche storico, delle carte costituzionali precedenti e della loro attualità.
\end{abstract}

Parole d'ordine: Doveri giuridici. Ordenamento giuridico. Costituzione italiana.

RESUMO: Este breve estudo pretende enfrentar o conceito de dever jurídico, analisando em particular no âmbito da teoria geral do direito. Partindo de um exame breve, abstrato e filosófico, de tal significado, tentamos analisar o dever como "dever constitucional", com referência particular para a Constituição italiana e fazendo um breve percurso histórico dos documentos precedentes constitucionais e de suas atualidades.

Paravras-chave: Deveres Jurídicos. Ordenamento Jurídico. Constituição Italiana.

SOMMARIO: Introduzione; 1 Cenni sul concetto di "dovere giuridico"; 2 I tratti comuni dei doveri fondamentali: tra filosofia e diritto; 3 I doveri nell'ordinamento costituzionale italiano: storia ed attualità; Bibliografia.

\section{INTRODUZIONE}

La manualistica giuridica tradizionale ha dedicato poca attenzione ai doveri, che invece, anche alla luce del XXI secolo, è necessario a nostro parere rivalutare ${ }^{1}$, proprio partendo dagli ascendenti filosofici e culturali che già sono stati affrontati e studiati in passato. Solo essendo consci dei propri doveri, infatti, ogni individuo potrà vivere con i propri diritti al sicuro, consapevole che ognuno di noi ha il dovere di rispettare i diritti degli altri e viceversa, affinché si possano creare le condizioni per una società più libera, giusta, e coesa. D'altronde, quando Immanuel Kant scriveva "agisci in modo da trattare l'umanità, sia nella tua persona sia in quella di ogni altro, sempre anche come fine e mai semplicemente come mezzo"2, non si riferiva, implicitamente al dovere?

\section{CENNI SUL CONCETTO DI "DOVERE GIURIDICO"}

Prima di cominciare un approfondimento dell'argomento sarà opportuno chiarire, almeno in via preliminare, il concetto di "dovere" nelle sue varie accezioni.

\footnotetext{
1 "Io voglio parlarvi dei vostri doveri. Voglio parlarvi, come il cuore mi detta, delle cose più sante che noi conosciamo." 1 Così si apre la celebre opera di Giuseppe Mazzini "dei doveri dell'uomo", un libro che sembra oggi uma bussola in questo mare magnum di aridità civica e culturale, che parte della società e della classe politica di oggi ci riserva quasi quotidianamente. Di G. MAZZINI, Dei doveri dell'uomo, 2010, RCS Libri, Milano, p. 17.

${ }^{2}$ I. KANT, Fondazione della metafisica dei costumi, in Scritti morali, traduzione di Pietro Chiodi, UTET, 1995 , pp. 88.
} 
Una magistrale definizione ci deriva proprio dalla filosofia kantiana ${ }^{3}$, che distingue tra doveri giuridici e doveri morali: Kant afferma che solo quelli che trovano la loro fonte nel diritto positivo possiedono la capacità d'imporsi, di essere eseguiti, anche contro la volontà di chi vi è tenuto (ossia in modo coattivo) attraverso l'esercizio del potere sanzionatorio; l'adempimento o la violazione dei doveri etici, viceversa, produce conseguenze solo nella sfera personale dell'obbligato e non è in grado di costituire il presupposto per l'attivazione di meccanismi sanzionatori. ${ }^{4}$

Il concetto di dovere, quindi, è insito nella stessa natura dell'uomo nel momento in cui si organizza in comunità sociale, e la differenza tra dovere morale e giuridico starà principalmente nella conseguenza del suo inadempimento o della sua violazione.

La stessa filosofia del diritto, secondo quanto sostenuto da parte della dottrina, è filosofia del dovere giuridico ${ }^{5}$, in quanto la vita del diritto non è solo quella della volontà particolare di "chi comanda", ma gli atti giuridici, e lo stesso ordinamento, sono prima di tutto un'idea universale che genera doveri necessari, e che esprimono anche il senso morale del diritto, poiché si determinano intorno alla funzione essenziale di quest'ultimo. Il dovere è il tema principale della norma giuridica.

Questo breve richiamo alla filosofia è necessario per porre la distinzione tra il dovere e l'obbligo, nonché tra il dovere inteso come " etico o morale" e dovere inteso come "giuridico", che in questo scritto si analizza nella sua specificità di "dovere costituzionale".

L'attenzione verso i doveri costituzionali infatti è essenziale, perché essi, come è stato osservato, hanno una straordinaria unicità': implicano un senso di appartenenza ad una comunità, e ciò è essenziale per l'unità politica; ed allo stesso tempo comportano il riconoscimento del prossimo, che è essenziale per la coesione sociale; come abbiamo precedentemente accennato, inoltre, essi portano all'adesione ad un sistema di valori condivisi, fondamentale per lo stesso ordine costituzionale democratico. L'adempimento dei doveri dà inoltre forma ad una comunità rispettosa dell'altro, nella quale i diritti possono essere serenamente esercitati.

Fra diritti e doveri esiste perciò una stretta interdipendenza, non solo per l'ovvia considerazione per la quale a ogni diritto di una persona corrisponde il dovere di un altro soggetto, ma perché i diritti, per essere tali, hanno bisogno anche del senso del dovere, ovvero del rispetto delle regole e dei principi morali; non per interesse o per timore della legge, ma semplicemente per l'obbligo che si ha nei confronti della propria coscienza. ${ }^{7}$

Infatti, come hanno sostenuto autorevoli autori, "noi non siamo liberi nonostante ai doveri, ma siamo liberi grazie ai doveri. Benché siano concetti affini, e siano spesso usati come sinonimi, i doveri sono differenti dagli obblighi. Mentre il dovere è un comando della nostra coscienza, l'obbligo è il comando di un 'autorità".

I doveri costituzionali contengono quindi questo pregio: di creare una commistione tra "obbligo" e "dovere", portando ad una vera responsabilizzazione etica del cittadino, che non è limitata al rispetto della legge in quanto obbligo generale ed astratto, ma che è rivolta ad un'osservanza pedagogica della legge fondamentale come principio di convivenza comune e civile. Senza una cultura ed una pratica dei doveri i cittadini si muovono come particelle isolate, perdono l'idea di appartenenza.

L'equilibrio tra diritti e doveri è dunque una delle principali virtù dei regimi democratici retti da una Costituzione ${ }^{9}$, ma purtroppo rappresenta anche il suo punto debole, perché, a differenza dei diritti, osservare i doveri richiede impegno, costanza e sacrificio. ${ }^{10}$

\section{I TRATTI COMUNI DEI DOVERI FONDAMENTALI: TRA FILOSOFIA E DIRITTO}

\footnotetext{
${ }^{3}$ I.KANT, La metafisica dei costumi, trad. it. 2004, LaTerza, Roma-Bari, pag 36 ss. Sostiene inoltre che "Mentre i doveri etici sono di obbligazione larga, quelli giuridici sono di stretta obbligazione." Al contrario, la coscienza; dell'individuo hobbesiano è tutt'altra da quella kantiana, ed anzi sono due concetti in opposizione. Per Hobbes l'individuo entrato in società sottomette la propria coscienza a quella del Leviatano, dal quale deriva poi il suo dovere; per Kant il dovere è invece il momento di universalizzazione della coscienza individuale.

${ }^{4}$ B. DE MARIA, "Etica repubblicana e Costituzione dei doveri”, 2013.

${ }^{5}$ A.INCAMPO Filosofia del dovere giuridico, 2014, Cacucci Editore, Bari, pag 9 ss. L'autore inoltre spiega l'interessante distinzione tra doveri "pragmatici" (se relativi ad atti) e tra doveri "sintattici" (se ordinamentali). Distingue inoltre tra "doveri di condotta" (che istituiscono rapporti giuridici) e "doveri di struttura" (che sono volti ad organizzare un corpus di norme). Non sarà possibile, in questa sede, soffermarsi ulteriormente su tale definizione. Ci permettiamo, però, di rimandare al testo.

${ }^{6}$ L.VIOLANTE, Il dovere di avere doveri, Giulio Einaudi Editore, Torino, 2014, p. 66.

${ }^{7}$ M.VIROLI, L'Italia dei doveri, RCS libri, Milano, 2008.

${ }^{8}$ Ibidem.

${ }^{9}$ L.VIOLANTE, Lettera ai giovani sulla Costituzione, 2006, Piemme Editore.

${ }^{10}$ S. MATTARELLI, Dialogo sui doveri, 2005, Marsilio Editori, Venezia, p. 19.
} 
Se partiamo da una visione generale ed introduttiva, notiamo che è sempre nella filosofia del diritto, prima ancora che nelle carte costituzionali scritte, che vengono individuati dei tratti comuni, una sorta di "genetica" dei doveri, che autorevoli autori ritengono siano caratterizzati da una "vocazione a dare un orientamento etico all'esperienza giuridica". ${ }^{11}$

In particolare ciò avviene nell'ambito dei doveri costituzionali, dove si delinea un vero e proprio anello di congiunzione tra etica e diritto.

Come è già stato sostenuto ${ }^{12}$, è solo dallo sforzo sinergico prodotto dai doveri che si può attendere un sostanziale ed apprezzabile appagamento dell'intera tavola dei valori costituzionali, e nella prospettiva dell'etica costituzionale diventa assai arduo separare i diritti dai doveri. ${ }^{13}$ Se ciò si fa, allora rischia di disperdersi il senso profondo e genuino di ciò che è la Costituzione stessa, svuotando di valore anche quella che dovrebbe essere la base dell'educazione civica: il rispetto della cosa pubblica.

Ma allora, una domanda sorge spontanea: qual è la ragione per cui i doveri costituzionali rappresentano un tema ancora oggi poco studiato ed approfondito? Una risposta è individuata da parte della dottrina in una ragione antecedente, individuabile nella circostanza che "nel divenire storico, il suddito, gravato proprio perché tale da una serie di doveri, trasformandosi in cittadino andava affermando via via i diritti" ${ }^{14}$, dimenticandosi (giustamente) dei doveri, che in quell'epoca storica erano obblighi imposti dai sovrani per imporre il proprio potere.

Tuttavia, come autorevole dottrina costituzionale ha puntualizzato ${ }^{15}$, per evitare fraintendimenti occorre "ribadire il nesso che i diritti intrattengono con l'esistenza dei doveri, e quindi di ricordare il tributo di onore che il linguaggio e la logica dei diritti devono portare al linguaggio e alla logica dei doveri". ${ }^{16}$

Infatti, mentre i doveri insistono sui legami che uniscono gli esseri umani, responsabilizzandoli, i diritti, se presi singolarmente in modo assoluto, rischiano di produrne piuttosto la separazione. Come è stato efficacemente sostenuto, "il punto principale della questione non riguarda il contenuto dei diritti o dei doveri, ma il movimento che essi producono: $i$ diritti isolano l'individuo, lo rinchiudono in una sfera privata, e anzi, lo contrappongono agli altri uomini; $i$ doveri invece lo costringono ad uscire da se stesso, portandolo immediatamente verso l'altro". ${ }^{17}$

Sul punto, illuminante è Guido Calogero, il quale scrisse: "che diritti mai avrebbero gli altri, se non sentissimo noi il dovere di riconoscerli, limitando perciò la nostra libertà con una norma o con una legge?" ${ }^{\text {. }}$. Altrettanto convincente è la tesi secondo cui i doveri, rispetto ai diritti, sono impopolari anche perché "essi sono confinati in una sorta di empireo morale, al limite tra la morale ed il diritto, e forse più vicini alla prima che non al secondo." 19

La citazione di Giuseppe Mazzini, fatta nelle note in prima pagina, non è casuale. Mazzini sosteneva infatti che la proclamazione dei diritti non aveva migliorato di molto la condizione del popolo, perché i diritti venivano riconosciuti agli individui senza munirli dei mezzi per esercitarli; si potrebbe obiettare che si è fatta molta strada da quando Mazzini scriveva, e che i diritti sociali hanno depotenziato quella obiezione. Ma qui, ovviamente, non

\footnotetext{
${ }^{11}$ A. RUGGERI, I doveri costituzionali: la prospettiva del giudice delle leggi: a cura di Balduzzi-Cavino-Grosso-Luther. Atti del convegno di Acqui termeAlessandria svoltosi il 9-10 giugno 2006. Giappichelli editore, Torino, p.566. L'autore sostiene che "tali doveri posseggono la capacità di proiettarsi fuori di sé, e, per ciò stesso, di proiettare fuori di sé l'intero ordinamento, ricongiungendosi con ciò che precede, determina, e dà senso alla Costituzione.

${ }^{12}$ A.RUGGERI, I doveri costituzionali: la prospettiva del giudice delle leggi: a cura di Balduzzi-Cavino-Grosso-Luther. Atti del convegno di Acqui termeAlessandria svoltosi il 9-10 giugno 2006. Giappichelli editore, Torino, p. 567.

${ }^{13}$ S. ROMANO, Principi di diritto costituzionale generale, 1947, Giuffré, Milano, pag 160. Teniamo a chiarire ulteriormente la differenza teorica tra diritti e doveri, riassunta dall'autorevole parere di Santi Romano: "mentre il diritto è signoria della volontà, il dovere è vincolo della volontà." Ecco perché entrambi debbono esistere (e coesistere) in una società che possa definirsi ben organizzata.

${ }^{14}$ G. LOMBARDI, I doveri costituzionali: la prospettiva del giudice delle leggi: a cura di Balduzzi-Cavino-Grosso- Luther. Atti del convegno di Acqui terme-Alessandria svoltosi il 9-10 giugno 2006. Giappichelli editore, Torino, p. 578 ss. L'autore prosegue sostenendo la sua tesi, dichiarando che "le grandi trasformazioni giuridiche e costituzionali si manifestano prima in termini di situazioni soggettive (i diritti sono una di queste) e solo dopo si traducono in forme organizzative delle istituzioni (e le forme di stato e di governo ne rappresentano il profilo principale) e si manifestano infine in una articolata distinzione delle fonti e della loro gerarchia: costituzione, leggi, atti di amministrazione, sentenze ecc, ne sono il prodotto.

${ }^{15}$ U. VINCENTI, "Prima il dovere, una ragionevole critica dei diritti umani". Jovene Editore, 2011.

${ }^{16}$ T. GRECO Prima il dovere, una critica della filosofia dei diritti: in "il senso della Repubblica" (a cura di S. MATTARELLI) pp. 2-3.

${ }^{17}$ T. GRECO Prima il dovere, una critica della filosofia dei diritti: in "il senso della Repubblica" (a cura di S. MATTARELLI) pp. 3-4..

${ }^{18}$ CALOGERO, Filosofia del dialogo, 1962, Edizioni di Comunità, Milano. pag 105 (citazione tratta da M. VIROLI, L'Italia dei doveri, p. 26.

${ }^{19}$ G. LOMBARDI, I doveri costituzionali: la prospettiva del giudice delle leggi: a cura di Balduzzi-Cavino-Grosso- Luther. Atti del convegno di Acqui terme-Alessandria svoltosi il 9-10 giugno 2006. Giappichelli editore, Torino, p. 579. L'autore procede ad una interessante disamina dell'influenza del pensiero mazziniano sull'evoluzione dele costituzioni post-liberali, sostenendo che "l'impostazione mazziniana rappresenta in un certo senso un superamento dell'originario modo di essere puramente liberale-liberistico delle costituzioni della borghesia, e proprio come tale esso abbia avuto scarso seguito sotto il profilo anche delle teorizzazioni costituzionali dell'Ottocento: è stato infatti con la fase costituzionale del primo dopoguerra che i doveri costituzionali sono entrati nei testi normativi." L'autore prossegue invitando allo studio dei doveri come "limite all'individualismo selvaggio basato su una asserita sovranità del mercato, quasi a fare dello stesso la nuova costituzione di uno Stato che si declassa a puramente strumentale".
} 
interessa ricostruire gli elementi della critica mazziniana ai diritti individuali, quanto semmai sottolinearne $\mathrm{i}$ principali elementi: i diritti individuali, infatti, sono incapaci di rendere conto di un elemento peculiare della natura umana, quale quello della socialità; sempre per riprendere le parole di Mazzini, l'individuo è sacro: i suoi interessi, i suoi diritti, sono inviolabili; ma porli come unico fondamento dell'edificio politico, e dire agli individui "conquisti ciascuno, e colle sole forze che ha, il proprio avvenire", è un dare la società e il progresso agli arbitri del caso e alle alternative di una lotta perenne, è un trascurare il fatto principale dell'umana natura, la socialità; è un impiantar l'egoismo nell'anima e ordinare per ultimo il dominio dei forti sui deboli, di quei che possiedono mezzi su quei che ne sono privi ${ }^{20}$. Mazzini non chiedeva di rinunciare ai diritti, indicava la via per conquistarli e goderli davvero, e la individuava nel dovere. Come scrisse lui stesso: "ogni vostro diritto non può essere frutto che d'un dovere compiuto: $i$ diritti non sono se non una conseguenza di doveri adempiuti." 21

Eminenti giuristi ${ }^{22}$, per tornare all'attualità, per quanto concerne il contenuto del dovere di solidarietà sancito all' art 2 della Costituzione (pietra angolare sulla quale sono costruiti gli altri doveri) ricollegano il suo fondamento proprio all'elaborazione teorica "dei doveri dell'uomo" mazziniana, dimostrando come questa abbia influito notevolmente sul dibattito svolto in Assemblea Costituente.

Questo ragionamento, in via teorica, è fondamentale per porre a noi stessi ed alla società attuale in cui viviamo, una domanda: quanto e come è cambiato di questa concezione? possiamo oggi dire che i numerosi diritti (anche sociali oltre che civili) contenuti nelle carte costituzionali siano veramente disponibili e concretamente esigibili da parte dei cittadini? Non sarà nostro compito, in questa sede, dare una risposta esaustiva; questo scritto è, piuttosto, un momento di riflessione condivisa, in cui noi giuristi (e studiosi di altre discipline umane), con spirito critico, coltiviamo il dubbio in modo da porci ulteriori domande.

\section{I DOVERI NELL'ORDINAMENTO COSTITUZIONALE ITALIANO: STORIA ED ATTUALITÀ}

Il richiamo al "dovere" non è cosa nuova nemmeno nel costituzionalismo scritto, e l'Italia, anche se originariamente divisa, ha avuto tante significative esperienze riguardo.

Per quel che riguarda la storia costituzionale italiana possiamo citare la Costituzione di Bologna del 1796, che si può considerare, almeno in via temporale, come prima Costituzione italiana (seppur non rigida e preunitaria). In essa troviamo una bellissima elencazione di doveri, di cui vale la pena trascrivere i più significativi: al suo art II, si legge che tutti i doveri dell'uomo e del cittadino derivano da questi due principi impressi dall'Autore della natura nel cuore dell'uomo - "Non fate ad altri quel che non vorreste fatto a Voi. - Fate in ogni occasione agli altri quel bene, che vorreste per Voi."

Tale principio è poi quasi totalmente riprodotto, seppur in prosa differente, anche nell'art. II della Costituzione della Repubblica Cisalpina del 1797: "Tutt'i doveri dell'uomo e del cittadino derivano da questi due principii scolpiti dalla natura in tutti i cuori. Non fate agli altri ciò che non vorreste fatto a voi. Fate costantemente agli altri il bene che vorreste fatto a voi".

Tornando alla Costituzione di Bologna del 1796, all'art III invece è sancito che I doveri di ciascun individuo verso la Società sono: difenderla, servirla, vivere soggetto alle leggi, e rispettare coloro che ne sono gli organi. All'art VI chi contravviene apertamente alle leggi si dichiara in stato di guerra con la Società ed all'art VII è sancito che chi senza contravvenire apertamente alle leggi, le elude con astuzia, offende il pubblico bene, e si rende indegno della comune stima e benevolenza. Più di 200 anni fa quindi il senso del bene comune era presente, ed ha caratterizzato tutte le Costituzioni che si sono susseguite.

Un'altra affascinante opera giuridica che vale la pena di prendere in considerazione, è la Costituzione del Popolo Ligure del 1797, che divide i "doveri dell'uomo in società" ed i "doveri del corpo sociale", e cioè una forma primordiale dei doveri dei cittadini e dei doveri dello Stato.

\footnotetext{
${ }^{20}$ G. Mazzini, Interessi e principii, in Edizione Nazionale degli scritti di Giuseppe Mazzini, Cooperativa tipografico-editrice Paolo Galeati, Imola (19061961), vol VIII, pp.175-176.

${ }^{21}$ Tratto da T. GRECO "Dai diritti al dovere: tra Mazzini e Calogero" in "Repubblicanesimo, democrazia, socialismo delle libertà: incroci per una rinnovata cultura politica" (a cura di T. CASADEI), FrancoAngeli Editore, 2004, p. 142-143.

${ }^{22}$ B. DE MARIA, "Etica repubblicana e Costituzione dei doveri". 2013, editoriale scientifica, Napoli, pp. 36-37. Sul punto, anche altri autori hanno evidenziato il richiamo alla teoria di Mazzini in Assemblea Costituente (in particolare il riferimento fu richiamato dal Padre Costituente Meuccio Ruini); Ad esempio A.PACE, in "I diritti fondamentali" (pubblicazione in "associazione italiana dei costituzionalisti") ci spiega che "il riferimento di Ruini a Mazzini è certamente di grande aiuto per intendere la genesi e il significato dell'art 2 Cost". (Citazione tratta da F. PIZZOLATO "Finalismo dello Stato e sistema dei diritti nella Costituzione italiana" Editore Vita \&amp; Pensiero, edizioni dell’Università Cattolica di Milano, 1999, pp. $209-210$.
} 
Sui doveri dell'uomo in società, si legge al suo art. I che "i diritti altrui sono il limite morale dei nostri e il principio dei doveri. Si adempiono questi col rispettare quelli. Il loro fondamento è in questa massima: fa costantemente agli altri il bene che vorresti riceverne. Non fare ad altri il male che non vorresti fatto a te stesso. "

Sui doveri del corpo sociale, invece, l'art 1 esplicita che il dovere della società verso gli individui che la compongono è la garanzia sociale: essa consiste nell'azione di tutti per assicurare i diritti di ciascheduno, dove essa manchi non vi è ne società né governo.

Ci sia permesso, in ultimo, di citare la Costituzione Napoletana del 1799, vero capolavoro illuministico, che non dà più una divisione bilaterale, ma una tripartizione in: doveri dell'uomo; doveri del cittadino; doveri dei pubblici funzionari. Essa sancisce anche dei veri e propri "precetti di umanità", come il "sacro dovere dell'uomo di alimentare i bisognosi" (art 19) e l'obbligo di ogni uomo di "illuminare e d'istruire gli altri" (art 20).

L'elenco dei doveri nella storia costituzionale italiana è molto ampio e particolarmente appassionante, in quanto possiamo contare 15 Costituzioni diverse. ${ }^{23}$

Non è possibile, in questo breve scritto, andare oltre nell'elencazione storica, ma cercherò, in conclusione, di soffermarmi sugli attuali doveri costituzionali.

Nella carta fondamentale del 1948, la forma di Stato Repubblicana, ovviamente delineata dai principi fondamentali della Costituzione italiana, pone al centro del rapporto tra autorità e libertà la tutela dei diritti inviolabili della persona umana, unitamente all'adempimento dei doveri inderogabili di solidarietà politica, economica e sociale affermati al suo art 2.

Questo, come già sostenuto da altri autori ${ }^{24}$, ha un significato molto profondo, perché non esprime solo un "rapporto" tra diritti inviolabili e doveri inderogabili, ma delinea un principio fondante dell'intero ordine repubblicano. Il ruolo svolto dalla nostra carta costituzionale rispetto ai doveri giuridici al suo interno individuati, quindi, non è solo descrittivo o precettivo, ma ha un fine: fare emergere i valori ed i principi supremi ${ }^{25}$ che hanno orientato proprio le scelte espresse in Assemblea Costituente, alla cui realizzazione sono tesi i doveri, espressamente o implicitamente posti da essa, in quanto "sul piano normativo, un imperativo che imponga una determinata condotta, non può che essere funzionale al perseguimento di uno scopo, il quale deve corrispondere a un valore. In questo caso, i valori della nostra Costituzione repubblicana." 26

Se analizziamo il "tessuto" dei doveri nella nostra Costituzione, notiamo che essi trovano le loro fondamenta nell'art 2, vero fulcro e pietra angolare dei doveri costituzionali. Il nesso tra diritti e doveri è un pilastro del nostro ordine costituzionale fissato proprio qui: tutto il sistema dei doveri costituzionali richiama il principio di unità politica, senza la quale i diritti diventano fogli di carta librati nel vuoto.

Tale norma sancisce che "La Repubblica riconosce e garantisce $i$ diritti inviolabili dell'uomo, sia come singolo sia nelle formazioni sociali ove si svolge la sua personalità, e richiede l'adempimento dei doveri inderogabili di solidarietà politica, economica e sociale." Da questa disposizione interpretiamo che essi, oltre alla classica divisione tra doveri delle persone e doveri delle istituzioni, si dividono principalmente in due categorie $^{27}$ : i doveri di solidarietà politica; i doveri di solidarietà economico-sociale.

Possiamo ora procedere ad una sintetica elencazione direttamente dal testo costituzionale:

L' Art 54 sancisce tre diversi doveri: il dovere di fedeltà alla Repubblica; il dovere di osservanza alla Costituzione e alle leggi; il dovere di obbedienza ${ }^{28}$ dei pubblici funzionari: "Tutti i cittadini hanno il dovere di essere fedeli alla

\footnotetext{
${ }^{23}$ Quella di Bologna del 1796, quella provvisoria del Piemonte del 1796, del popolo ligure del 1797, della repubblica Cisalpina del 1797 e della repubblica Cispadana del 1797, quella napoletana del 1799, quelle del Regno di Sicilia del 1812 e del Regno di Napoli del 1815 e del Regno delle due sicile del 1820 ; Lo Statuto Albertino del 1848, lo Statuto del granducato di toscana del 1848, il progetto di Costituzione di Antonio Rosmini del 1848, la nostra amata Costituzione della Repubblica Romana del 1849 e la Carta del Carnaro del 1920, per poi arrivare alla Costituzione della Repubblica italiana del 1947-48.

${ }^{24}$ B. DE MARIA, "Etica repubblicana e Costituzione dei doveri”. 2013, editoriale scientifica, Napoli.

${ }^{25}$ Questo è rilevato dichiaratamente da P. CARETTI ed E.CHELI, in "L'influenza dei valori costituzionali sui sitemi giuridici contemporanei" (a cura di A.Pizzorusso e V. Varano) in cui i due autorevoli autori sostengono che è indubitabile che ogni epoca storica abbia conosciuto l'influenza di certi valori costituzionali, dotati di una straordinaria forza espansiva, in virtù della quale essi hanno condizionato in misura maggiore o minore gli sviluppi di più ordinamenti giuridici".

${ }^{26}$ B. DE MARIA, "Etica repubblicana e Costituzione dei doveri”. 2013, editoriale scientifica, Napoli, p. 4-5.

${ }^{27}$ G. LOMBARDI, "Contributo allo studio dei doveri costituzionali”. 1967, Giuffrè Editore, Milano.

${ }^{28}$ La distinzione teorica tra dovere di fedeltà, dovere di osservanza e dovere di obbedienza, è particolarmente complessa e non è il caso, in questa sede, di procedere ad una sua analisi. Sul punto, però, ci sia permesso di riportare quella parte della dottrina costituzionalistica che ne ha evidenziato i tratti differenziali. In particolare, G.TREVES in "Fondamento giuridico del dovere di fedeltà dei funzionari ed impiegati pubblici" (in "studi sassaresi", 1933, vol XII, pag 428) osserva che mentre il dovere di fedeltà e quello di osservanza sono condizionati dalla sola esistenza dela norma che li stabilisce, quello di obbedienza abbisogna di due norme: una prima che lo stabilisce, una seconda (l'ordine del superiore) che sulla base della prima rende possibile - con la determinazione dell'oggetto, altrimenti insussistente - l'obbedienza. Anche G. LOMBARDI, in "Contributo allo studio dei doveri costituzionali". 1967, Giuffrè Editore, Milano, pag 120 ss scrive che Il testo costituzionale non nomina espressamente l'obbedienza, ma la presuppone (...) ed è necessario tener
} 
Repubblica e di osservarne la Costituzione e le leggi. I cittadini cui sono affidate funzioni pubbliche hanno il dovere di adempierle con disciplina ed onore, prestando giuramento nei casi stabiliti dalla legge."

L' Art 52 sancisce il dovere di difesa della Patria ${ }^{29}$ e il dovere di prestazione militare (poi trasformato in dovere di servizio civile e poi, purtroppo, sospeso): "La difesa della Patria è sacro dovere del cittadino. Il servizio militare è obbligatorio nei limiti e nei modi stabiliti dalla legge."

L'Art 48 riguarda il dovere di voto. ${ }^{30}$ Il voto è personale ed eguale, libero e segreto. Il suo esercizio è dovere civico.

Riguardo ai doveri di solidarietà economica e sociale invece, essi sono: Il dovere tributario all'art 53: Tutti sono tenuti a concorrere alle spese pubbliche in ragione della loro capacità contributiva. Il sistema tributario è informato ai criteri di progressività. ${ }^{31}$

Il dovere di istruzione all'art $30^{32}$ : È dovere e diritto dei genitori mantenere, istruire ed educare i figli, anche se nati fuori del matrimonio. Nei casi di incapacità dei genitori, la legge provvede a che siano assolti i loro compiti.

I doveri di attività e lavoro ${ }^{33}$ all'art 4: Ogni cittadino ha il dovere di svolgere, secondo le proprie possibilità e la propria scelta, un'attività o una funzione che concorra al progresso materiale o spirituale della società.

Anche l'art. 112 Cost. può considerarsi come impositivo di un dovere, che impone al Pubblico Ministero (magistrato inquirente) l'obbligo di esercitare l'azione penale, corollario dell'eguaglianza dei cittadini di fronte alla legge.

nettamente distinto l'osservanza delle leggi dal dovere di obbedienza. Già il carattere relazionale dell'obbedienza fa avvertire la necessità della distinzione: l'ordine, dal quale l'obbedienza deriva, trae infatti, nel sistema della legalità statuale, la sua ragione giustificatrice proprio dalla sua conformità alla legge e quindi riposa esso stesso sul dovere di osservanza, mentre a sua volta l'obbedienza è l'atto terminale in cui l'osservanza delle leggi si esaurisce. Ancora, l'autore prosegue spiegando che mentre l'osservanza si collega logicamente a norme giuridiche, la fedeltà si riporta a valori istituzionalmente desumibili dalla Costituzione. Si potrebbe dire che oggetto della fedeltà è il contenuto materiale di una certa Costituzione, assunto come sua ragione leggittimatrice, mentre oggetto dell'osservanza sono le norme di un testo costituzionale. pp. 146-147.

${ }^{29}$ Questo dovere, è definito come il dovere più alto che la Costituzione ci indica. "Definendolo come sacro" i Padri Costituenti vollero metterne in evidenza il significato religioso: religioso non perché comandato dal Dio della rivelazione, ma perché il dovere di difendere la Patria può esigere anche il sacrificio della vita, e la vita può sacrificarla soltanto una persona che ha una concezione religiosa. Per chi non l'ha, la parola "sacro" non ha un senso, e un "sacro dovere" suona alle sue orecchie come una facezia o come una esagerazione retorica. Condividiamo con questa analisi, alla quale rimandiamo per un approfondimento, in 29 M. VIROLI, L'Italia dei doveri, RCS libri, Milano, 2008, p 73-74. Sul punto, G. LOMBARDI, in "Contributo allo studio dei doveri costituzionali". 1967, Giuffrè Editore, Milano, p.243 ci spiega che strettamente legato al dovere di difesa, ma distinto sul piano concettuale e normativo, è il dovere di prestazione militare. Esso infatti rappresenta un profulo strumentale e organizzativo del dovere di difesa, ma allo stesso tempo se ne distingue perché limitato nei mezzi e circoscritto. Inoltre, mentre il dovere di difesa grava su tutti i cittadini, il dovere di prestazione militare grava soltanto sui soggetti individuati come idonei alla prestazione alle armi. Bisogna evidenziare, tuttavia, che l'autore scrisse questa monografia in un momento storico in cui la leva militare era ancora presente. Oggi, come sappiamo, è stata sospesa. Si può quindi sostenere che il dovere di difesa si può e si deve configurare anche come un sacro "dovere civile". "Oggetto della difesa" dunque non sarà solo lo Stato-Nazione come entità territoriale, ma il principio democratico che informa lo Stato stesso, e come tale, la difesa della Costituzione repubblicana e dei suoi valori.

${ }^{30}$ In Assemblea Costituente venne abbandonata la dizione originaria secondo la quale il voto, oltreché "dovere civico" veniva qualificato come "dovere morale", e parallelamente si rinunciò ad un emendamento aggiuntivo per cui il voto poteva "essere reso obbligatorio dalla legge". L'on Mortati si era espresso in favore della sola dizione di "dovere morale", affermando che tuttavia esso avrebbe dovuto intendersi "non nel senso che la Costituzione crei doveri morali, bensi' nel senso che, richiamandoli, rafforzi nel cittadino la convinzione di qualcosa che investe la qualità e la funzione morale dell'uomo" (Atti Assemblea Costituente, p 4133). Interessante lo spunto di G. LOMBARDI, in “Contributo allo studio dei doveri costituzionali”. 1967, Giuffrè Editore, Milano, pag 339 ss in cui sostiene che il dovere di voto non solo si inquadra perfettamente nel sistema postulato dal principio di sovranità popolare sancito dall'art 1 comma secondo della Costituzione, ma consente di ravvisare nel dovere di voto un dovere costituzionale in senso stretto, che rappresenta l'anello di congiunzione tra il momento individualistico e il principio di solidarietà politica, intesa come partecipazione alla determinazione delle supreme decisioni statuali.

${ }^{31}$ Non sarà inutile evidenziare l'importanza del dovere di "pagare le tasse". Il dovere tributario infatti (definito anche "dovere fiscale" o "dovere contributivo") è l'antecedente logico e necessario della vita dello Stato, e conseguentemente è garante per l'adempimento di tutti gli altri diritti che la Costituzione garantisce e prevede. Senza contribuzione, lo Stato come entità giuridica non esiste. Crediamo che oggi sia necessario considerare la tassazione come il prezzo che il contribuente paga per vivere in una società civile (Sono parole del giudice della Corte Suprema Oliver Wendell Holmes Jr., citate in A. CATTARIN in "Dalla servitù alla sovranità. No taxation without representation”, 2009, Jovene, Napoli, cap II par 10); Inoltre, ci teniamo a ribadire la concezione gobettiana secondo cui "In Italia il contribuente non ha mai sentito la sua dignità di partecipe alla vita statale:la garanzia del controllo parlamentare sulle imposte non era un'esigenza, ma una formalità giuridica. Il contribuente italiana paga bestemmiando lo Stato; esso non ha la coscienza di esercitare, pagando, una vera e propria funzione sovrana. L'imposta gli è imposta. In P. GOBETTI, La rivoluzione liberale, 1924. Saggio sulla lotta politica in Italia, Grandi Tascabili Economici Newton, Roma, 1998, p.179. Sulla progressività delle imposte non è opportuno, in questa sede, proseguire ulteriormente.

${ }^{32}$ In realtà, le disposizioni costituzionali in materia di cultura e istruzione emergono non solo dall'art 30 , ma dal suo combinato disposto con gli art 33 e 34 Cost. Anche se vengono espressi termini differenti (insegnamento, istruzione, scuola) essi si muovono in affini ambiti concettuali. Dall'esame delle norme, emerge come sia distinto il "momento pubblicistico" dal "momento privatistico" di tale dovere.

${ }^{33}$ La norma richiamata (art 4 secondo comma) è un vero e proprio fondamento costituzionale degli obblighi di prestazione e di collaborazione civica. In Assemblea Costituente venne respinto un emendamento volto ad elevare l'adempimento del dovere di attività a condizione per l'esercizio dei diritti politici (sul punto rimandiamo a "La Costituzione della Repubblica Italiana illustrata con i lavori preparatori, pag 27 ss" di Falzone-Palermo-Cosentino); tuttavia bisogna chiarire che il legislatore non potrà configurare una sola prestazione omogenea ai fini dela realizzazione del dovere di attività, ma dovrà garantire, appunto, una libertà di scelta, come chiaramente si evince dalla disposizione normativa. Inoltre, non è corretto parlare solamente di "dovere di lavoro", ma di vero e proprio "dovere di collaborazione civica", intesa come estrinsecazione del dovere di attività nel normale fluire della vita sociale, politica, ed economica. 
Da questo lavoro emerge che i doveri costituzionali sono costruiti sul perno di tre nuclei inscindibili: quello della solidarietà politica, quello economico, e quello sociale; è su questi che è enucleato il concetto di doveri fondamentali. Essi infatti non possono considerarsi come tre ambiti categoriali distinti, ma vivono attraverso una rete di reciproche interferenze, nelle quali il cittadino (e lo Stato) sono tenuti ad operare e ad adempiere ai doveri che ne conseguono.

Questa constatazione porta ad una ulteriore riflessione: il fondamento dei doveri costituzionali è, prima di tutto, un fondamento personalistico ${ }^{34}$, basato sul senso di responsabilità dell'individuo nei confronti della comunità politico-sociale. Il concetto di dovere costituzionale, quindi, non è collegabile al concetto di "supremazia dello Stato", ma anzi, è proprio nei doveri che viene limitato il potere pubblico nella sfera giuridica individuale. I diritti fondamentali da un lato, e i doveri costituzionali dall'altro, segnano una rivalutazione della persona che viene in tal modo il nucleo centrale della Costituzione repubblicana.

\section{BIBLIOGRAFIA}

A.CATTARIN in "Dalla servitù alla sovranità. No taxation without representation”, 2009, Jovene, Napoli A.INCAMPO Filosofia del dovere giuridico, 2014, Cacucci Editore, Bari.

A. RUGGERI I doveri costituzionali: la prospettiva del giudice delle leggi: a cura di Balduzzi-Cavino-Grosso-Luther. Atti del convegno di Acqui terme-Alessandria svoltosi il 9-10 giugno 2006. Giappichelli editore, Torino.

B. DE MARIA, “Etica repubblicana e Costituzione dei doveri”. 2013, editoriale scientifica, Napoli.

F.PIZZOLATO "Finalismo dello Stato e sistema dei diritti nella Costituzione italiana" Editore Vita \& Pensiero, edizioni dell’Università Cattolica di Milano, 1999.

G. CALOGERO, Filosofia del dialogo, 1962, Edizioni di Comunità, Milano.

G. MAZZINI, Interessi e principii, in Edizione Nazionale degli scritti di Giuseppe Mazzini, Cooperativa tipografico-editrice Paolo Galeati, Imola (1906-1961), vol VIII.

G. LOMBARDI, "Contributo allo studio dei doveri costituzionali”. 1967, Giuffré Editore, Milano.

G. MAZZINI, Dei doveri dell'uomo, 2010, RCS Libri, Milano,

I. KANT, La metafisica dei costumi, trad. it. 2004, LaTerza, Roma-Bari.

I. KANT, Fondazione della metafisica dei costumi, in Scritti morali, traduzione di Pietro Chiodi, UTET, 1995.

L. VIOLANTE, Il dovere di avere doveri, Giulio Einaudi Editore, Torino, 2014.

L. VIOLANTE, Lettera ai giovani sulla Costituzione, 2006, Piemme Editore.

M. VIROLI, L'Italia dei doveri, RCS libri, Milano, 2008.

N. OCCHIOCUPO, Liberazione e promozione umana nella Costituzione: unità di valori nella pluralità di posizioni, 1995 , Giuffrè Editore, Milano.

P.GOBETTI, La rivoluzione liberale, 1924. Saggio sulla lotta politica in Italia, Grandi Tascabili Economici Newton, Roma, 1998.

S. ROMANO, Principi di diritto costituzionale generale, 1947, Giuffré, Milano.

S.MATTARELLI, Dialogo sui doveri, 2005, Marsilio Editori, Venezia.

T.GRECO "Dai diritti al dovere: tra Mazzini e Calogero" in "Repubblicanesimo, democrazia, socialismo delle libertà: incroci per una rinnovata cultura politica" (a cura di T.CASADEI), FrancoAngeli Editore, Milano, 2004.

T.GRECO Prima il dovere, una critica della filosofia dei diritti: in "il senso della Repubblica" (a cura di S.MATTARELLI) Franco Angeli Editore, Milano, 2006.

U. VINCENTI, Prima il dovere: una ragionevole critica dei diritti umani, Jovene, 2011.

\footnotetext{
${ }^{34}$ Sul concetto di "fondamento personalistico" ci sia permesso di rimandare alla bellissima opera di N. OCCHIOCUPO, Liberazione e promozione umana nella Costituzione: unità di valori nella pluralità di posizioni, 1995, Giuffrè Editore, Milano.
} 